\title{
Experimental Research of Induction Heating in Inner Hollow of Cylindrical Solenoid
}

\author{
Viktoriia Strelnikova
}

\begin{abstract}
Purpose: The experimental research of magnetic field strength distribution and induced current density in tubular metal blank depending on its position inside cylindrical inductor. Methodology: Electrodynamics problem solution using mathematical methods for analytical calculations. Expressions for fields and currents flowing in non-magnetic metals under a multi-turn cylindrical solenoid influence have been derived from Maxwell's equations system. The accepted mathematical assumptions and simplifications do not affect the correctness of the results. Conducting the experiment to determine magnetic field strength inside solenoid hollow and induced currents density on surface of non-magnetic metal billet. Results: The obtained experimental distributions of magnetic field strength and induced currents when a billet is completely placed inside cylindrical inductor hollow correspond to the previously derived analytical relations and coincide with the fundamental physical concepts of the ongoing processes in the system. With partial placement of the billet inside the inductor, there is an abrupt increase in the tangential magnetic field strength. An extremum is observed in the end zone of a billet in this case. This is due to induced currents concentration in this area as well as on cylindrical surface of a billet. Scientific novelty. Average induction heating velocities correspond to the calculated values for the materials of the processed samples used in the experiment. Thus it testifies to the effectiveness of using this processing technology equally, both non-magnetic metals and magnetic. The practical significance of the results lies in the possibility of assessing the qualitative indicators of the level of induced currents and thermal effects in the end parts of non-magnetic cylindrical blanks with partial overlapping by an inductor for performing operations.
\end{abstract}

Index Terms-Cylindrical Solenoid, Eddy Currents, Heating Speed, Maxwell's Equations.

\section{INTRODUCTION}

Induction heating is being used in many industries, but for repair aims it mostly used in automobile industry. Depending on the configuration of inductor-tool there can be conducted different technological operations such as flattening of dents on car bodies (external non-contact alignment) or taking off stickers and glasses etc. [1], [2]. This paper presents the consideration of characteristics of the tool typically intended for bolt joints reconnection, extrusion of different clamp types, dismantling of complex structures units which can't be brought to desired form by traditional methods without losing its of functionality. Induction heating of such type allows local and quick destroy the corrosion layer on details, which in many cases

Published on August 31, 2020.

V. Strelnikova, Kharkiv National Automobile and Highway University, Ukraine.

(e-mail: v.strelnikova91@gmail,com) caused the complications during listed operations performance.

Today many engineering solutions based on the description of similar electrodynamic problem, but they cannot be called universal [3], [4]. For this reason, in the development of high-efficiency inductor systems, the key stages are a complete analytical review of a specific unsolved problem and conducting an experiment in the laboratory, in order to identify the main factors that influence on the obtaining of the desired result.

In the design of the induction heating equipment, in particular tool-inductor, there can be distinguished two intersecting problems. The first one is problem with achievement of simultaneous processing throughout the whole metal billet, which is often the case of the multipurpose use of induction heating systems. It leads to second one - mathematical complications in drawing up an adequate model without introducing physical assumptions that distort it.

The purpose of this paper is the experimental determination of the magnetic field strength and the density of induced currents distribution dependences in the metal of the tubular blank from its position in the internal hollow of the cylindrical inductor.

\section{Formulation of the Problem}

A conditional move of the tubular blank along the cylindrical inductor central axis (Fig.1) holds the preservation of azimuthal symmetry.

Despite this, the displacement of the blank in the direction that does not satisfy the condition $\left(l / R_{1}\right)>>1$, leads to the inhomogeneity of the magnetic field and the appearance of edge effects in the end zone of the tubular billet. In the future, this will entail a complication of the mathematical model, because of the need to introduce a component describing these edge effects.

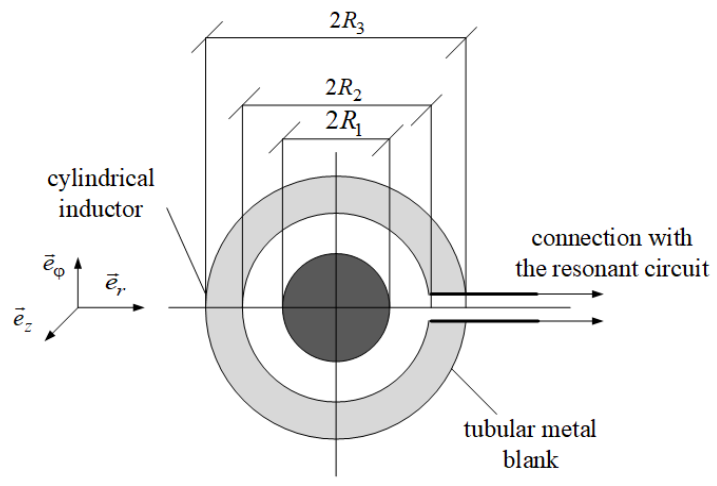

(a) 


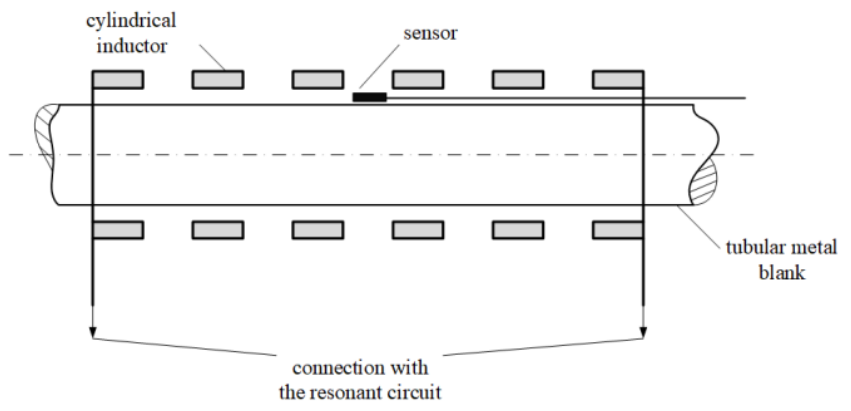

(b)

Fig. 1. Multi-turn cylindrical solenoid: a - cross-sectional view and radial dimensions of the solenoid and blank; $b$ - longitudinal section view of the solenoid and the blank, the location of the field strength sensor inside the structure

The experiment was dedicated to determination of magnetic field strength in axis direction of its inner hollow and superficial distribution of induced currents in metal billet with its different longitudinal placement in the inductor. Of particular practical and physical interest is the behavior of these parameters on the end surface of the billet.

\section{Calculation Model}

Consider the basic mathematical model of the process of induction heating of tubular blanks, described in [5].

There are main assumptions that have been used for the experiment planning:

- the presence of a gap between wires leading to power installation can be neglected and it can be supposed that there is circular symmetry when $\frac{\partial}{\partial \varphi}=0(\varphi$ is the azimuthal angle);

- the energy of the excited magnetic field is concentrated in the inner hollow of the solenoid and on its inner surface, the longitudinal z-component of the intensity will be equal to: $H_{i}(t)=\frac{W \cdot J_{i}(t)}{l_{i}}$ where $t$ is the time, $W$ is the number of turns, $J_{i}(t)$ is the exciting current, $l_{i}$ is the length of the inductor winding;

- the condition of quasi-stationarity is acceptable, so that $\omega \cdot \frac{a}{c}<<1$ where $\omega$ is the characteristic cyclic frequency in the spectrum of the exciting current, $a$ is the characteristic size of the system, $c$ is the speed of light in vacuum.

From Maxwell's system of differential equations transformed by Laplace [6], [7], can be obtained equation for electric field strength in different environments with different electrophysical characteristics:

$$
\frac{\partial}{\partial r} \cdot\left(\frac{1}{r} \cdot \frac{\partial}{\partial r}\left(r \cdot E_{\varphi}(p, r)\right)\right)-k_{1,2}^{2}(p) \cdot E_{\varphi}(p, r)=0
$$

where $k_{1,2}(p)$ - wave numbers,

$$
k_{1,2}(p)=\left\{\begin{array}{l}
k_{1}(p)=\frac{p}{c}-\text { for spaces } \\
\text { with dielectric filling; } \\
c-\text { the speed of light; } \\
k_{2}(p)=\sqrt{p \mu_{0} \gamma}-\text { for spaces } \\
\text { with the ultimate conductivity. }
\end{array}\right.
$$

\section{$p$ - Laplace's transform parameter \\ $\mu$ - magnetic permeability of blank metal \\ $\gamma$ - conductivity of blank metal}

The fundamental solution system of (1) is Bessel's modified functions of the first order $-I_{1}(z), K_{1}(z)$ [7].

According to this for non-zero field components (in cylindrical coordinate system with guiding orts $\vec{e}_{r}, \vec{e}_{\varphi}, \vec{e}_{z}$ ) $H_{z, r} \neq 0$ и $E_{\varphi} \neq 0$ obtained next dependences:

- for electric field strength

a) in the air gap between the inner surface of the inductor winding and the outer surface of the blank, $r \in\left[R_{1}, R_{2}\right]$ :

$E_{\varphi}^{(1)}(p, r)=A(p) \cdot I_{1}\left(k_{1}(p) \cdot r\right)+B(p) \cdot K_{1}\left(k_{1}(p) \cdot r\right)(2)$

b) in the blank metal, $r \in\left[0, R_{1}\right]$ :

$$
E_{\varphi}^{(2)}(p, r)=C(p) \cdot I_{1}\left(k_{2}(p) \cdot r\right)
$$

where $A(p), B(p), C(p)$ - arbitrary constants of integration.

The arbitrary constants of integration in (2) and (3) can be found using corresponding boundary conditions.

- $\quad$ for magnetic field strength

$$
\begin{aligned}
& H_{z}^{(2)}(p, r)=-\left(\frac{Z_{0} \cdot \gamma \cdot W \cdot R_{1}}{l}\right) \times \\
& \times \frac{J_{i}(p)}{\left(k_{1}(p) \cdot R_{1}\right)\left(k_{2}(p) \cdot R_{1}\right)} \cdot \frac{1}{\Delta(p)} \cdot I_{0}\left(k_{2}(p) \cdot r\right)
\end{aligned}
$$

where $W$ - winding turns number of exciting solenoid; $Z_{0}$ - wave resistance of vacuum.

Assuming that the induced current flows along the azimuth in a solid cylindrical blank of radius $-R_{1}$ and length $-l$, can be find the L-image of $J(\mathrm{p})$ by the law of total current:

$$
\begin{aligned}
& J(p) \approx l \cdot\left(H_{z}^{(2)}(p, 0)-H_{z}^{(2)}\left(p, R_{1}\right)\right)= \\
& =\frac{\left(W \cdot Z_{0} \cdot \gamma \cdot R_{1}\right)}{\left(\left(k_{2}(p) \cdot R_{1}\right) \cdot\left(k_{1}(p) \cdot R_{1}\right)\right)} \times \\
& \times J_{i}(p) \cdot \frac{\left(I_{0}\left(k_{2}(p) \cdot R_{1}\right)-1\right)}{\Delta(p)}
\end{aligned}
$$


The equation (5) can be simplified if it takes into account the actual conditions for performing repair operations that require induction heating of the working objects.

After necessary identical transformations in (5) obtained the following

$$
\begin{aligned}
& J(p) \approx W \cdot J_{i}(p) \times \\
& \times \frac{\left(1-\sqrt{2 \pi \cdot\left(k_{2}(p) R_{1}\right)} \cdot \exp \left(-k_{2}(p) R_{1}\right)\right)}{\left(\frac{\left(k_{1}(p) R_{1}\right) \cdot\left(k_{2}(p) R_{2}\right)}{\left(Z_{0} \gamma R_{1}\right)} \cdot\left(\frac{R_{1}}{R_{2}}\right) \cdot \ln \left(\frac{R_{1}}{R_{2}}\right)-1\right)}
\end{aligned}
$$

As the expression (6) contains uncertainties, opening them, in the original space can be obtained a directly proportional relationship between the exciting current of the solenoid and the current induced in the metal of the heated cylindrical billet.

$$
J(t) \approx W \cdot J_{i}(t)
$$

The result (7) indicates that the magnitude of the induced current in cylindrical induction heating systems with working frequencies about 30 to $50 \mathrm{kHz}$ and steel nonmagnetic blanks (about $0.4 \cdot 10^{7} \quad 1 / \mathrm{Om} \cdot \mathrm{m}$ ) with crosssectional dimensions about 0.01 to $0.05 \mathrm{~m}$ is set exclusively by the winding turns number of the exciting solenoid $-W$ and doesn't depend either on temporal characteristics of electromagnetic processes - $\omega$ or on system geometry determined by ratio $-\left(R_{1} / R_{2}\right)$ [5].

\section{Determination OF THE Distribution PARAMETERS OF INDUCED CURRENTS}

Experimental studies were carried out on the equipment of the laboratory of electromagnetic technologies Kharkiv National Automobile and Highway University.

Schematic diagram of the induction heating system includes the following components: power source, converter-inductor, blank and induction sensor, which measures the values of the estimated indicators.

\section{A. Technical characteristics of the model:}

1. Solenoid geometry: length $-0.052 \mathrm{~m}$, radius -0.02 $\mathrm{m}$;

2. Turns number -20 ;

3. Magnitude of the source current - 15 A (when obtaining the field distribution) - $30 \mathrm{~A}$ (when determining the blank heating temperature);

4. Ampere-turns, $x=\mathrm{W} \cdot \mathrm{J}, \mathrm{x} \in[100,1000]$;

5. Cross-sectional area $S \approx \delta \cdot l$, where $\delta$ - value of the skin-layer, $\delta=\sqrt{\frac{2}{\omega \cdot \gamma \cdot \mu_{0}}}$, and length about $2 \pi R_{l}$.

Because the wavelength is greater than the penetration depth the tubular blank assumed as solid, therefore, the thickness of the walls is not taken into account in the calculations [8].

6. Inductor working frequency $\omega=30 \mathrm{kHz}$.

\section{B. Heating objects:}

Tubular blanks of L62 brass (diamagnetic, based on the properties of the copper alloy as the main component) and aluminum (paramagnetic).

\section{Progress of the experiment:}

Proceeding from the statement of the problem, the tubular blank was placed as follows: $100 \%, 75 \%, 50 \%$ and $25 \%$ of the blank length in the inner inductor hollow.

As shown in Fig.1b the induction sensor was placed between the inductor and the tubular blank. It was decided to measure the magnetic field strength at 10 points with an offset step $\Delta x=5 \mathrm{~mm}$, for this purpose, using an electronic oscilloscope, the readings of the EMF were recorded in $\mathrm{mV}$ for each of the four blank positions.

Further, using Faraday's law (8) for the sensor and knowing that the strength of the magnetic field varies according to the harmonic law obtained the following dependences:

$$
\varepsilon_{i}=-\frac{d \Phi}{d t}=-S \cdot N \frac{d B}{d t}=-S \cdot N \cdot \mu \frac{d H}{d t}
$$

where $S$ - sensor's area, $\mathrm{S}=1 \cdot 10^{-6} \mathrm{~m}^{2}$;

$$
N \text { - sensor's turns number, } N=100 \text {. }
$$

$$
\begin{gathered}
H(t)=H_{m} \sin (\omega t) \\
H_{m}=\frac{\varepsilon_{m}}{S N \mu \omega}=\frac{1}{S N \mu \omega} \cdot \varepsilon_{m}=k \cdot \varepsilon_{m}
\end{gathered}
$$

Because the experiment was carried out using high frequencies at which the field does not penetrate through the blank, the statement $H_{m i}=j_{i}$, is true, then the current induced in the blank will be numerically equal to:

$$
J=\int_{0}^{\infty} j d x=\sum_{i} j_{i} \Delta x
$$

where $j_{i}$ - induced current density

Induced currents density distributions in relative units for different blank materials are showed in Fig.2 and Fig.3.

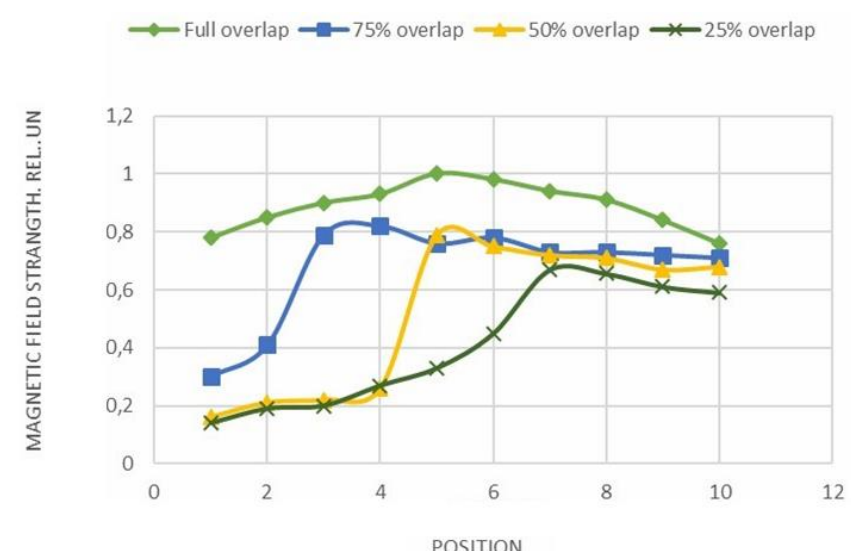

Fig. 2. Induced currents distribution in aluminum in units normalized to a maximum 


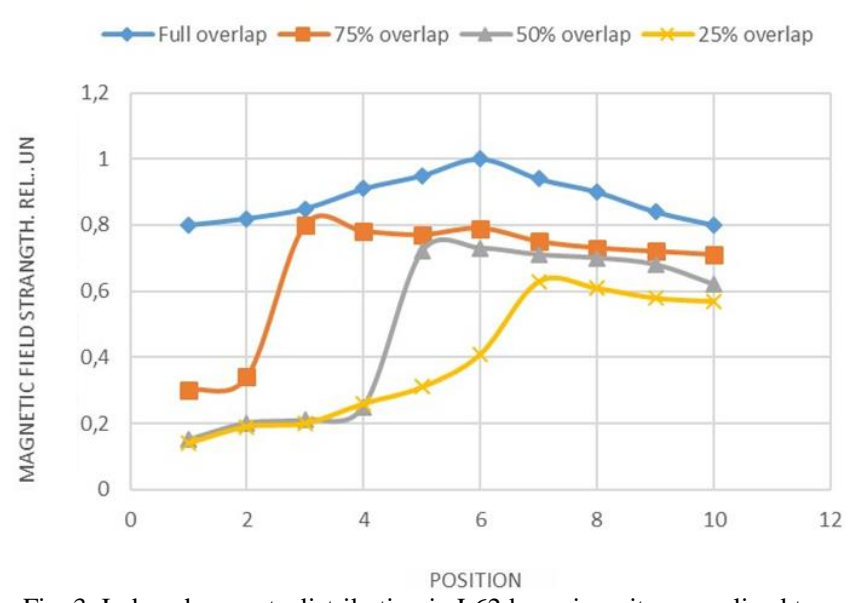

Fig. 3. Induced currents distribution in L62 brass in units normalized to a maximum

By (9), obtained the following values of induced current in blanks (on the maximum values $-100 \%$ of the blank length in the inductor hollow for aluminum and for L62 brass):

$$
\begin{aligned}
& -\quad \text { aluminum } \mathrm{J}=201.48 \mathrm{~A} \\
& -\quad \text { L62 brass } \mathrm{J}=197.51 \mathrm{~A}
\end{aligned}
$$

\section{BLANK HEATING}

The flow of eddy currents is accompanied by the release of Lenz-Joule heat [6]. Suppose that in the absence of heat transfer heating occurs only in that part of the blank where the eddy currents are concentrated. We give numerical estimates of the heating carried out under laboratory conditions, using the dependence derived in [5]. The indicator of this evaluation is the dynamic characteristic of the process - the average induction heating speed:

$$
\bar{V}=\left(\Delta T^{\circ} / \Delta t\right)
$$

Accepted heating time $\Delta t=30 \mathrm{~s}$, which corresponds to condition $\Delta t \gg 2 \pi / \omega$. Values $\Delta T^{\circ}$ obtained using pyrometer.

Omitting the intermediate transformations for the materials taken in the experiment, the following values of the average heating speed were obtained:

$$
\begin{array}{ll}
- & \text { aluminum } \bar{V}=0,19{ }^{\circ} \mathrm{C} / \mathrm{s} \\
- & \text { L62 brass } \bar{V}=0,24{ }^{\circ} \mathrm{C} / \mathrm{s}
\end{array}
$$

\section{CONCLUSION}

The main results of the study include the following:

1. The obtained experimental distributions of the magnetic field strength and induced currents with the complete placement of the blank in the cylindrical inductor hollow correspond to the analytic relations derived earlier and coincide with the fundamental physical concepts of the proceeding processes.

2. It is shown that the magnitude of the induced current of this idealization for cylindrical induction heating systems is established solely by the winding turns number of the exciting solenoid and doesn't depend either on the temporal characteristics of the electromagnetic processes or on the system geometry.

3. When the blank is partially placed in the inductor inner hollow, there is an abrupt increase in the tangential magnetic field strength. In this case, an extremum is observed in the end zone of the blank. This is due to the concentration of induced currents in this area, as well as on the cylindrical surface of the billet.

4. Average heating velocities correspond to the calculated values for the processed samples materials used in the experiment, which indicates the efficiency of induction heating both of non-magnetic metals and magnetic ones.

\section{REFERENCES}

[1] [Online] http://www.josam-ua.com/indukcionnie_nagrevateli.html

[2] [Online] http://www.redhotdot.ru/Product.aspx?vP/D=11943

[3] A. Slukhotskiy, Induction heating installations. Leningrad: Energoizdat Publ., 1981, 328 p.

[4] Yu. Batygin, and V. Lavinskiy, "Features of magnetic-pulsed "distribution" of thin-walled tubular billets", Elektrichestvo Electricity, no. 11, pp. 62-68, 2005.

[5] Yu. Batygin, Ye. Chaplygin, O. Sabokar, and V. Strelnikova, "Induction heating in the inner cavity of the cylindrical solenoid. Running processes main relationships", Herald of Khmelnytskyi national university, no. 6, pp. 62-68., 2017.

[6] H. Kuhling, Dictionary on Physics. Translated from German. Moscow: Mir Publ., 1982, 520 p.

[7] I. N. Bronshteyn, and K. A. Semendyayev, Mathematical handbook for engineers and university students. Moscow: Nauka Publ.. 1986, $723 \mathrm{p}$.

[8] Yu. Batygin, A. Gnatov, I. Trunova, and Ye. Chaplygin, Ye. (2011) Progressive technologies for vehicles. Part 2. Physical fundamentals of magnetic-pulsed technologies of non-contact alignment of car body elements: handbook. Kharkov: KHNADU Publ., 2011, 176 p.

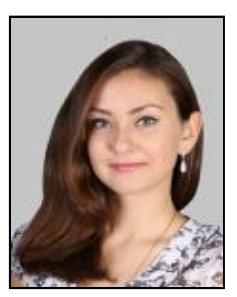

Strelnikova Viktoriia Anatolyiivna born in 1991, Kharkiv, Ukraine. In 2014 completed Master's degree course in Transportation technologies and got a Diploma with Honors of Kharkiv National Automobile and Highway University.

Currently postgraduate student and Junior researcher of the Laboratory of electromagnetic technologies, Kharkov National Automobile and Highway University.

The main direction of scientific activity is the solution of theoretical and practical problems:

- research of the influence of magnetic properties of structural elements of inductors on the efficiency of induction heating;

- development of a new scientific direction associated with the "direct passage of current" through the object of processing during the performance of operations on the magnetic-pulse attraction of thin-walled metals. 